\title{
Assessment of localisation to auditory stimulation in post-comatose states: use the patient's own name
}

\author{
Lijuan Cheng $^{1 \dagger}$, Olivia Gosseries ${ }^{2 \dagger}$, Limei Ying ${ }^{1}$, Xiaohua $\mathrm{Hu}^{3}$, Dan Yư ${ }^{3}$, Hongxing Gao ${ }^{3}$, Minhui He ${ }^{1}$,
} Caroline Schnakers ${ }^{2}$, Steven Laureys ${ }^{2}$ and Haibo $\mathrm{Di}^{1,2^{*}}$

\begin{abstract}
Background: At present, there is no consensus on how to clinically assess localisation to sound in patients recovering from coma. We here studied auditory localisation using the patient's own name as compared to a meaningless sound (i.e., ringing bell).

Methods: Eighty-six post-comatose patients diagnosed with a vegetative state/unresponsive wakefulness syndrome or a minimally conscious state were prospectively included. Localisation of auditory stimulation (i.e., head or eyes orientation toward the sound) was assessed using the patient's own name as compared to a ringing bell. Statistical analyses used binomial testing with bonferroni correction for multiple comparisons.
\end{abstract}

Results: 37 (43\%) out of the 86 studied patients showed localisation to auditory stimulation. More patients ( $n=34,40 \%)$ oriented the head or eyes to their own name as compared to sound ( $n=20,23 \% ; p<0.001$ ).

Conclusions: When assessing auditory function in disorders of consciousness, using the patient's own name is here shown to be more suitable to elicit a response as compared to neutral sound.

Keywords: Localisation to sound, Auditory localisation, Vegetative state, Unresponsive wakefulness syndrome, Minimally conscious state, Own name, Disorders of consciousness

\section{Background}

At present, there is no consensus on what auditory stimulus should be employed for the assessment of localisation to sound in disorders of consciousness such as the "vegetative state" (now also coined unresponsive wakefulness syndrome; VS/UWS [1]) and the minimally conscious state (MCS) [2]. Indeed, several behavioural "coma scales" use different stimuli to evaluate auditory localisation. For instance, the Coma Recovery Scale-Revised (CRS-R), the Sensory Modality Assessment Rehabilitation Technique and the Western Neuro-Sensory Stimulation Profile leave the choice open between several auditory stimuli (e.g., noise, voice). The Coma/Near Coma Scale requests to use

\footnotetext{
*Correspondence: dihaibo@yahoo.com.cn

${ }^{\dagger}$ Equal contributors

'International Vegetative State and Consciousness Science Institute, Hangzhou Normal University, Hangzhou, China

${ }^{2}$ Coma Science Group, Cyclotron Research Centre and Neurology

Department, University and University Hospital of Liege, Liege, Belgium Full list of author information is available at the end of the article
}

" $5 \mathrm{~s}$ of bell ringing", and the Wessex Head Injury Matrix uses a noise (bell, whistle or buzzer) and "a person talking" (for a review, see [3]).

We here propose to use the patient's own name (as compared to a meaningless noise) in the assessment of localisation to sound. The own name is intrinsically meaningful for each of us because of its personal significance, emotional content and repetition along life. Beyond our day-to-day experience, the extreme salience of being presented one's own name was highlighted in various experimental and clinical studies. Some of these suggest that self-referential stimuli are so potent that they can "capture attention and subsequently bring the stimulus into awareness" [4]. In everyday social interactions, auto-referential stimuli give rise to a sense of self-awareness, as reflected in the cocktail party phenomenon when hearing our own name [5]. This particularly easy detection in usual laboratory experiments with healthy participants is consistent with research that showed powerful detection of the own 
Table 1 Auditory localisation according to diagnosis and aetiology

\begin{tabular}{|c|c|c|c|c|c|c|c|}
\hline \multirow[t]{2}{*}{ Diagnosis } & \multicolumn{4}{|c|}{ Localisation response } & \multicolumn{2}{|c|}{ Aetiology } & \multirow[t]{2}{*}{ Tota } \\
\hline & Own name & Bell & Both & None & Traumatic & Non traumatic & \\
\hline VS/UWS & $4(5 \%)$ & $1(1 \%)$ & $4(5 \%)$ & $38(44 \%)$ & $26(30 \%)$ & $21(25 \%)$ & 47 \\
\hline MCS & $13(15 \%)$ & $2(2 \%)$ & $13(15 \%)$ & $11(13 \%)$ & $27(31 \%)$ & $12(14 \%)$ & 39 \\
\hline Total & $17(20 \%)$ & $3(3 \%)$ & 17 (20\%) & 49 (57\%) & $53(61 \%)$ & $33(39 \%)$ & 86 \\
\hline
\end{tabular}

Number of patients showing localisation to the own name, ringing bell or both as a function of diagnosis [vegetative state (VS/UWS) versus minimally conscious state (MCS)] and aetiology.

name in situations of reduced consciousness [6,7]. The aim of the present study is to determine whether the assessment of localisation to sound in patients recovering from coma is influenced by the choice of the auditory stimulus.

\section{Methods}

Eighty-six patients recovering from coma were prospectively assessed free of sedative drugs. Each patient was studied in a sitting position and a standardized arousal facilitation protocol (i.e., deep pressure stimulations from the facial musculature to the toes) was employed if needed in order to prolong the length of time the patient maintained arousal [8]. Localisation to sound was evaluated using a standardized methodology as described in the CRS-R [8]. In brief, an auditory stimulus (bell and patient's own name) was presented from the right and from the left side while the examiner stood next to the patient but out of view. Stimuli were matched for intensity and duration of presentation, and were presented twice for each side. The order of presentation was randomized using "random number" procedure in Excel. Localisation to auditory stimulation was defined as head or eyes orientation toward the location of the stimulus on both trials for at least one side. Special care was made not to present stimuli when spontaneous eye or head movements were occurring. Clinical diagnosis was made according to the Aspen workgroup criteria for

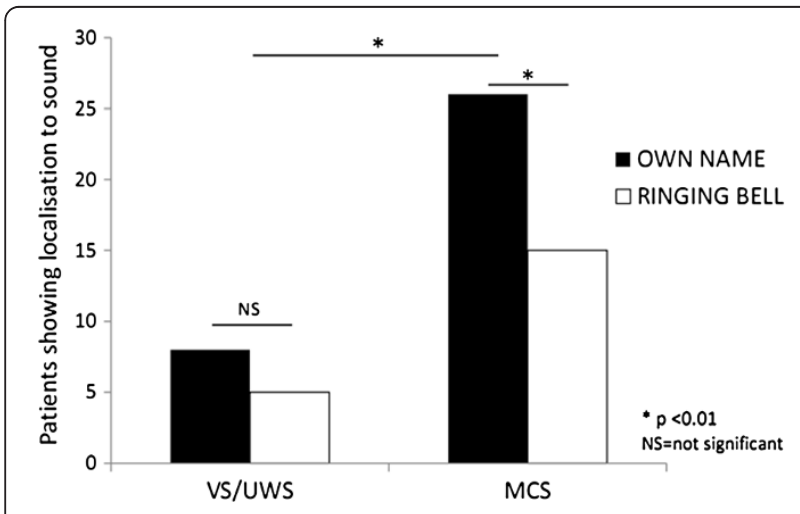

Figure 1 Auditory localisation. Number of patients in vegetative/ unresponsive state (VS/UWS) and minimally conscious state (MCS) showing localisation to sound $(n=37)$ as a function of the employed stimulus (own name in black and ringing bell in white). disorders of consciousness [2] and based on the CRS-R assessments [8] performed by two trained and experienced neuropsychologists. Note that according to these guidelines auditory localisation is compatible with the diagnosis of VS/UWS. The study was approved by the Ethics Committee of Hangzhou Normal University and Wujing Hospital which complies with the Code of Ethics of the World Medical Association (Declaration of Helsinki). Informed consents were obtained by the patient's legal surrogates.

Differences between localisation as assessed by bell or patient's own name were measured using binomial testing (Stata Statistical Software; Release 11.2. College Station, TX: StataCorp LP 2009). Bonferroni correction was applied for multiple comparisons. Results were considered significant at $\mathrm{p}<0.01$.

\section{Results}

Out of 86 patients (67 men; mean age 46 (SD 17) years), 47 (55\%) were diagnosed in VS/UWS [1] and 37 (45\%) were in MCS. Median time between injury and assessment was 5 months (IQR: 3 - 13 months). Aetiology was traumatic in $53(61 \%)$ and non-traumatic in 33 (39\%) patients. 37 (43\%) out of the 86 studied patients showed localisation to auditory stimulation. Overall, more patients $(n=34,40 \%)$ oriented the head or eyes to the own name as compared to sound $(n=20,23 \%$; $\mathrm{p}<0.001$ ) (Table 1). MCS patients localized more often to their own name as compared to sound $(\mathrm{p}<0.001)$. This effect was not significant in the VS/UWS group $(\mathrm{p}>0.05)$ (Figure 1).

Tables 2 and 3 show the clinical data for each patient (MCS and VS/UWS patient groups respectively). Localisation preference was not different depending on aetiology or time since insult $(\mathrm{p}>0.05)$. The overall behavioural responsiveness assessed by the CRS-R total score tended to be higher when patients localized both stimuli than when they did not show any localisation (Tables 2 and 3). For instance, MCS patients showing both responses to their own name and to the bell had a CRS-R total score between 9 and 18 whereas MCS patients showing no localisation had a score between 6 and 10. Patients localizing to their own name only (or bell only) showed intermediate CRS-R total scores. In the 37 patients showing localisation, 9 patients were considered 
Table 2 Clinical data of the MCS patients

\begin{tabular}{|c|c|c|c|c|c|}
\hline Patient & Gender & Aetiology & Time since injury* & CRS-R score ${ }^{* *}$ & Auditory localisation \\
\hline MCS1 & male & trauma & 7 & 6 & none \\
\hline MCS2 & male & trauma & 73 & 7 & none \\
\hline MCS3 & male & trauma & 21 & 7 & none \\
\hline MCS4 & female & trauma & 20 & 7 & none \\
\hline MCS5 & male & trauma & 155 & 8 & none \\
\hline MCS6 & female & trauma & 160 & 8 & none \\
\hline MCS7 & female & trauma & 21 & 8 & none \\
\hline MCS8 & male & trauma & 205 & 9 & none \\
\hline MCS9 & male & trauma & 20 & 10 & none \\
\hline MCS10 & male & stroke & 51 & 10 & none \\
\hline MCS11 & male & trauma & 45 & 10 & none \\
\hline MCS12 & female & trauma & 9 & 8 & bell \\
\hline MCS13 & male & stroke & 32 & 9 & bell \\
\hline MCS14 & male & trauma & 55 & 8 & own name \\
\hline MCS15 & male & trauma & 11 & 8 & own name \\
\hline MCS16 & male & trauma & 221 & 8 & own name \\
\hline MCS17 & male & trauma & 150 & 9 & own name \\
\hline MCS18 & male & trauma & 40 & 9 & own name \\
\hline MCS19 & female & stroke & 14 & 9 & own name \\
\hline MCS20 & male & stroke & 61 & 10 & own name \\
\hline MCS21 & male & trauma & 22 & 10 & own name \\
\hline MCS22 & male & trauma & 19 & 13 & own name \\
\hline MCS23 & male & trauma & 7 & 13 & own name \\
\hline MCS24 & female & trauma & 54 & 14 & own name \\
\hline MCS25 & male & trauma & 291 & 14 & own name \\
\hline MCS26 & male & stroke & 115 & 16 & own name \\
\hline MCS27 & male & anoxia & 50 & 9 & both \\
\hline MCS28 & female & trauma & 7 & 10 & both \\
\hline MCS29 & male & trauma & 13 & 10 & both \\
\hline MCS30 & male & trauma & 121 & 10 & both \\
\hline MCS31 & male & trauma & 33 & 11 & both \\
\hline MCS32 & male & stroke & 13 & 11 & both \\
\hline MCS33 & male & trauma & 12 & 12 & both \\
\hline MCS34 & male & stroke & 9 & 13 & both \\
\hline MCS35 & female & stroke & 22 & 15 & both \\
\hline MCS36 & male & anoxia & 135 & 16 & both \\
\hline MCS37 & male & stroke & 6 & 16 & both \\
\hline MCS38 & male & anoxia & 57 & 17 & both \\
\hline MCS39 & male & trauma & 3 & 18 & both \\
\hline
\end{tabular}

*Time since injury in weeks, ** Total score of the Coma Recovery Scale-Revised (minimum 0, maximum 23). 
Table 3 Clinical data of the VS/UWS patients

\begin{tabular}{|c|c|c|c|c|c|}
\hline Patient & Gender & Aetiology & Time since injury* & CRS-R score ${ }^{* *}$ & Auditory localisation \\
\hline VS/UWS1 & male & stroke & 9 & 2 & none \\
\hline VS/UWS2 & male & anoxic & 6 & 3 & none \\
\hline VS/UWS3 & male & trauma & 34 & 3 & none \\
\hline VS/UWS4 & male & trauma & 17 & 3 & none \\
\hline VS/UWS5 & male & stroke & 66 & 4 & none \\
\hline VS/UWS6 & male & anoxic & 82 & 4 & none \\
\hline VS/UWS7 & male & trauma & 39 & 4 & none \\
\hline VS/UWS8 & male & trauma & 13 & 4 & none \\
\hline VS/UWS9 & female & anoxia & 4 & 4 & none \\
\hline VS/UWS10 & male & stroke & 13 & 5 & none \\
\hline VS/UWS11 & male & anoxia & 89 & 5 & none \\
\hline VS/UWS12 & male & stroke & 5 & 5 & none \\
\hline VS/UWS13 & female & trauma & 8 & 5 & none \\
\hline VS/UWS14 & male & trauma & 189 & 5 & none \\
\hline VS/UWS15 & female & stroke & 5 & 5 & none \\
\hline VS/UWS16 & male & stroke & 3 & 5 & none \\
\hline VS/UWS17 & female & trauma & 68 & 6 & none \\
\hline VS/UWS18 & male & trauma & 76 & 6 & none \\
\hline VS/UWS19 & male & trauma & 36 & 6 & none \\
\hline VS/UWS20 & male & trauma & 13 & 6 & none \\
\hline VS/UWS21 & male & trauma & 22 & 6 & none \\
\hline VS/UWS22 & male & trauma & 8 & 6 & none \\
\hline VS/UWS23 & male & stroke & 21 & 6 & none \\
\hline VS/UWS24 & male & trauma & 9 & 6 & none \\
\hline VS/UWS25 & male & stroke & 16 & 6 & none \\
\hline VS/UWS26 & male & trauma & 34 & 6 & none \\
\hline VS/UWS27 & male & trauma & 19 & 6 & none \\
\hline VS/UWS28 & male & trauma & 12 & 6 & none \\
\hline VS/UWS29 & female & stroke & 11 & 6 & none \\
\hline VS/UWS30 & male & trauma & 70 & 6 & none \\
\hline VS/UWS31 & male & anoxia & 413 & 6 & none \\
\hline VS/UWS32 & male & trauma & 28 & 6 & none \\
\hline VS/UWS33 & male & trauma & 34 & 7 & none \\
\hline VS/UWS34 & male & trauma & 24 & 7 & none \\
\hline VS/UWS35 & female & trauma & 11 & 7 & none \\
\hline VS/UWS36 & male & stroke & 9 & 7 & none \\
\hline VS/UWS37 & female & stroke & 13 & 7 & none \\
\hline VS/UWS38 & male & trauma & 10 & 7 & none \\
\hline VS/UWS39 & female & anoxic & 16 & 4 & bell \\
\hline VS/UWS40 & male & anoxic & 557 & 8 & bell \\
\hline VS/UWS41 & male & stroke & 39 & 5 & own name \\
\hline VS/UWS42 & female & trauma & 18 & 6 & own name \\
\hline VS/UWS43 & male & anoxia & 23 & 7 & own name \\
\hline VS/UWS44 & female & anoxia & 15 & 7 & own name \\
\hline
\end{tabular}


Table 3 Clinical data of the VS/UWS patients (Continued)

\begin{tabular}{|c|c|c|c|c|c|}
\hline VS/UWS45 & female & trauma & 20 & 6 & both \\
\hline VS/UWS46 & male & trauma & 14 & 7 & both \\
\hline VS/UWS47 & male & trauma & 38 & 8 & both \\
\hline
\end{tabular}

*Time since injury in weeks, **Total score of the Coma Recovery Scale-Revised (minimum 0, maximum 23).

as being in VS/UWS according to the CRS-R criteria (i.e., they showed no response to command, no orientation to pain and no visual tracking) - 4 of these patients showed orientation to the own name but not to sound.

\section{Discussion}

Our data show that the assessment of localisation to sound depends on what stimulus is employed. MCS patients tend to best orient to their own name as compared to a meaningless loud sound (i.e., ringing bell). Indeed, one's own name is a piece of information that we use to process in the auditory modality from infancy: $4-5$ month-old infants are able to recognize the sound pattern of their own names [9]. In end-stage demented patients, it has also been shown that perception of the own name deteriorates well after perception of time, place and recognition [10]. Similarly, after general anaesthesia, the patient's reactivity to the own name occurs first, before reactivity to pain and noise [11]. In MCS patients, clinical experience learns that behavioural responses to auto-referential stimuli such as the own face are amidst the first signs heralding further recovery of consciousness [12]. Eventrelated potential studies have also shown that hearing one's own name, as compared to meaningless noise, leads to an increased mismatch negativity response in patients with disorders of consciousness [6]. In addition, functional MRI studies assessing brain activation to the own name have reported activation of "self"-related brain regions (i.e., anterior cingulate and mesiofrontal cortices) depending of the level of consciousness in patients recovering from coma $[7,13]$.

$28 \%$ of the studied MCS patients (11/39) failed to show auditory localisation. Neurological assessment showed that 2 of these 11 patients (18\%) had absent auditory startle, while 9 (82\%) showed auditoryindependent signs of consciousness. In line with previous studies, auditory impairment probably explains this finding [3].

Auditory localisation seems to be related to the patient's overall behavioural responsivity: the more the patients are conscious, the more they tend to respond to both auditory stimuli. Moreover, our results showed that most of the patients who responded to the bell also responded to their own name (condition "both" in Table 1). Three patients however showed localisation to the bell but not to their own name. Even if they retained basic auditory processing, these three patients might not have been able to process language, and hence recognize their own name. Another explanation could be the presentation of the patient's own name as last stimulus, and hence fatigue might explain orientation to a bell in the absence of orientation to the own name.

One should note that the duration and the degree of the movement towards auditory stimulation were not taken into account to assess auditory localisation (as described in the CRS-R). This should nevertheless be investigated in future studies to allow differentiating between a brief movement and a sustained fixation following auditory stimulation. Indeed, the latter may potentially be considered as a sign of consciousness, as it is the case for visual and tactile localisation (e.g., visual pursuit and localisation to noxious stimulation items in the CRS-R). Such responses may also be worth exploring further using neuroimaging techniques such as fMRI and EEG in order to compare the behavioral responses and the underlying cerebral networks involved when hearing the person's name being called.

\section{Conclusions}

Our findings emphasize the clinical importance of using the patient's own name when performing bedside testing of localisation to sound, adding to previous studies the importance of using auto-referential stimuli in patients with disorders of consciousness (i.e., the use of a mirror in the assessment of visual tracking [12]).

\section{Abbreviations}

VS/UWS: Vegetative state/unresponsive wakefulness syndrome;

MCS: Minimally conscious state; CRS-R: Coma recovery scale-revised;

fMRI: Functional magnetic resonance imaging; EEG: Electroencephalography.

\section{Competing interests}

The authors declare that they have no competing interests.

\section{Authors' contributions}

LC, OG, LY, XU, DY and HG collected data and managed the patients; LC and OG performed data analyses; SL and HD designed the study; LC, OG, SL and $\mathrm{HD}$ wrote the paper. $\mathrm{CS}$ and $\mathrm{MH}$ revised the manuscript for important intellectual content. LC and OG contributed equally to the study. All authors read and approved the final manuscript.

\section{Acknowledgements}

This study was supported by the National Natural Science Foundation of China (30870861), the Science and Technology Department of Zhejiang Province (2008C14098), the Hangzhou Normal University and HNUEYT, the Belgian Funds for Scientific Research (FNRS), Fonds Léon Frédericq, and the University of Liège. The authors thank Cunlai Xu, Jian Gao, Kehong Liu, Kun Li,Yu Zhang, Hongyan Song and Yan Dong for their assistance in patients' assessment, and Didier Ledoux for his statistical advices. OG and CS are postdoctoral researchers and SL is research director at FNRS. 


\section{Author details}

'International Vegetative State and Consciousness Science Institute, Hangzhou Normal University, Hangzhou, China. ${ }^{2}$ Coma Science Group, Cyclotron Research Centre and Neurology Department, University and University Hospital of Liege, Liege, Belgium. ${ }^{3}$ Rehabilitation Center for Brain Damage, Wujing Hospital of Hangzhou City, Hangzhou, China.

Received: 7 December 2012 Accepted: 11 March 2013

Published: 18 March 2013

\section{References}

1. Laureys S, Celesia GG, Cohadon F, Lavrijsen J, León-Carrión J, Sannita WG, Sazbon L, Schmutzhard E, von Wild KR, Zeman A, Dolce G, European Task Force on Disorders of Consciousness: Unresponsive wakefulness syndrome: a new name for the vegetative state or apallic syndrome. BMC Med 2010, 8:68.

2. Giacino JT, Ashwal S, Childs N, Cranford R, Jennett B, Katz DI, Kelly JP, Rosenberg JH, Whyte J, Zafonte RD, Zasler ND: The minimally conscious state: Definition and diagnostic criteria. Neurology 2002, 58(3):349-53.

3. Majerus S, Gill-Thwaites H, Andrews K, Laureys S: Behavioral evaluation of consciousness in severe brain damage. Prog Brain Res 2005, 150:397-413.

4. Mack A, Pappas Z, Silverman M, Gay R: What we see: inattention and the capture of attention by meaning. Conscious Cogn 2002, 11(4):488-506.

5. Wood N, Cowan N: The cocktail party phenomenon revisited: How frequent are attention shifts to one's own name in an irrelevant auditory channel. J Exp Psychol Learn Mem Cogn 1995, 21:255-60.

6. Qin P, Di H, Yan X, Yu S, Yu D, Laureys S, Weng X: Mismatch negativity to the patient's own name in chronic disorders of consciousness. Neurosci Lett 2008, 448:24-8.

7. Qin P, Di H, Liu Y, Yu S, Gong Q, Duncan N, Weng X, Laureys S, Northoff G: Anterior cingulate activity and the self in disorders of consciousness. Hum Brain Mapp 2010, 31(12):1993-2002.

8. Giacino JT, Kalmar K, Whyte J: The JFK Coma Recovery Scale-Revised: measurement characteristics and diagnostic utility. Arch Phys Med Rehabil 2004, 85(12):2020-9.

9. Mandel DR, Jusczyck PW, Pisoni DB: Infants' recognition of the sound pattern of their own names. Psychol Sci 1995, 6:314-10.

10. Fishback DB: Mental status questionnaire for organic brain syndrome, with a new visual counting test. J Am Geriatr Soc 1977, 25(4):167-70

11. Kurtz D, Trapp C, Kieny MT, Wassmer JM, Mugnaioni MD, Pack A, Hoff E: Study of recovery and the post-anaesthetic period. Rev Electroencephalogr Neurophysiol Clin 1977, 7(1):62-9.

12. Vanhaudenhuyse A, Schnakers C, Bredart S, Laureys S: Assessment of visual pursuit in post-comatose states: use a mirror. J Neurol Neurosurg Psychiatry 2008, 79(2):223.

13. Di H, Yu SM, Weng XC, Laureys S, Yu D, Li JQ, Qin PM, Zhu YH, Zhang SZ, Chen YZ: Cerebral response to patient's own name in the vegetative and minimally conscious states. Neurology 2007, 68(12):895-9.

doi:10.1186/1471-2377-13-27

Cite this article as: Cheng et al:: Assessment of localisation to auditory stimulation in post-comatose states: use the patient's own name. BMC Neurology 2013 13:27.

\section{Submit your next manuscript to BioMed Central and take full advantage of:}

- Convenient online submission

- Thorough peer review

- No space constraints or color figure charges

- Immediate publication on acceptance

- Inclusion in PubMed, CAS, Scopus and Google Scholar

- Research which is freely available for redistribution 